\title{
An Ontology-based Approach to Semantic Health Resource Knowledge Base Development for Crisis Preparation Decision Support System
}

\author{
Min Zhu \\ Institute of Public Safety Research(IPSR), Tsinghua \\ University. \\ Navy General Hospital of PLA \\ Beijing, China \\ Ruxue Chen (co-first author) \\ Navy General Hospital of PLA \\ Beijing, China \\ 13381207330@126.com \\ Quanyi Huang \\ Institute of Public Safety Research(IPSR), Tsinghua \\ University. \\ Beijing, China \\ qyhuang@tsinghua.edu.cn
}

\begin{abstract}
Emergent health resource supply is the key support to the accomplishment of medical rescue mission. It contains huge data of various kinds of health resource to deal with. Soeme researches were directed to solve such problem, including using various optimization models (OM) and decision support system(DSS). However, these methods were not widely used. One major impediment is the lack of standard knowledge representation models to guide the knowledgebase development. Our research presents a new approach to develop a semantic health resource knowledge base (SHRKB), based on a semantic health resource knowledge ontology (SHRKO) using HL7 RIM to represent emergent medical rescue domain knowledge. We also build semantic health resource expression repository (SHRER) and queries encoded in SPARQL format to extend the SHRKO with dynamic decision logic. The method is validated in a case study of the medical assistance mission executed by the hospital ship Peace Ark. The results show that SHRKB could support same decision method come to more effective plan than normal knowledge base. Future, a more efficient DSS for crisis health resource preparation could be established based on the SHRKB.
\end{abstract}

Keywords- ontology; semantic; knowledge base; decision support; crisis preparation.

\section{INTRODUCTION \& RELATED WORK}

Emergent health resource supply is the key problem for curing people wounded in the unexpected crisis. Some research showed that the mortality and the disability rate had a linear relationship with the time when starting to treat the wounded. ${ }^{[1,2]}$ So, the emergent health resource should be prepared as quickly as possible to support the curing zhum14@mails.tsinghua.edu.cn

\author{
Jia Qu (co-first author) \\ Navy General Hospital of PLA \\ Beijing, China \\ vzcg6000@126.com
}

\author{
Xinzhi Wang \\ Institute of Public Safety Research(IPSR), Tsinghua \\ University. \\ Beijing, China \\ wxz15@mails.tsinghua.edu.cn
}

\author{
Shaobo Zhong* (corresponding author) \\ Institute of Public Safety Research(IPSR), Tsinghua \\ University. \\ Beijing, China \\ zhongshaobo@tsinghua.edu.cn*
}

treatment. However, the preparation is very complex because huge data of various types of health resources should be dealt with and several factors should be taken into concern. Different types of casualty would occur and hundreds types of medicine and medical equipments. There were many researches on the emergency supply management. Major researches were on four aspects, the selecting of location for storing ${ }^{[3,4]}$, the distribution ${ }^{[5,6]}$, the scheduling ${ }^{[7]}$ and the supplement ${ }^{[8]}$. Optimization models $(\mathrm{OM})$ and decision support systems (DSS) had become increasingly used in these researches to facilitate the management of emergency supply $[9,10,11]$. OM and DSS could substantially improve the supply effectiveness. However, despite its great potential and a history of successful cases to this day, DSS have not found widely use in medical rescue tasks outside of one or two medical maneuvers. One of the major impediments to its widespread adoption is the lack of standard semantic based knowledge representation models to guide the development of the knowledge base, which is the core component of a DSS for medical rescue tasks.

The ontology, originally defined as "a formal, explicit specification of a shared conceptualization" [12], has been considered as a key technology in knowledge engineering. Medical is a complicated domain. Compared with the data in other domains, there are too many natural language descriptions and terminologies in medical data. As a result, a variety of ontologies have been constructed in medical domain, for example, the Biocaster ontology [13], Unified 
Medical Language System (UMLS) Semantic Network System(Unified Medical Language System Semantic Network Documentation $)^{[14]}$, etc. On one hand, because of the distributed nature of ontology development, the proliferation of medical ontologies often have a feature of heterogeneity and overlapping domains. On the other hand, little research has been conducted on the rescue medical resource ontologies development. Both would obstacle to knowledge integration in emergent medical rescue domain. The ontologies we constructed in our research also made use of general medical domain ontologies already existing to describe general medical knowledge.

Our research presents a new approach to develop a semantic health resource knowledge base (SHRKB), based on a semantic health resource knowledge ontology (SHRKO) using Health Level Seven International (HL7) reference information model (RIM) to represent emergent medical rescue domain knowledge. We also build a semantic health resource expression repository (SHRER) of rules encoded in Jena rule format to extend the SHRKO with dynamic decision logic. The proposed method is validated in a case study of the overseas humanitarian medical assistance mission, to demonstrate its technical feasibility.

\section{TASK DESCRIPTION}

The methods and steps used in our research are as follows. First, we set the target domain and acquire the domain knowledge. Second, we use HL7 RIM to present emergent medical rescue domain knowledge. HL7 all message specifications are based on the RIM, a shared information model for integrating entity data and medical domain knowledge[15], which can serve as the basis of unified domain ontology development. We extract the core concepts, properties, attributes and links of the knowledge. Then we use top-down design pattern to develop ontologies. Third, we use web ontology language (OWL) to describe the meta ontology and add ontology instances to form the ontology base. Fourth, we build a semantic health resource expression repository (SHRER) of rules encoded in Jena rule format. Finally, we

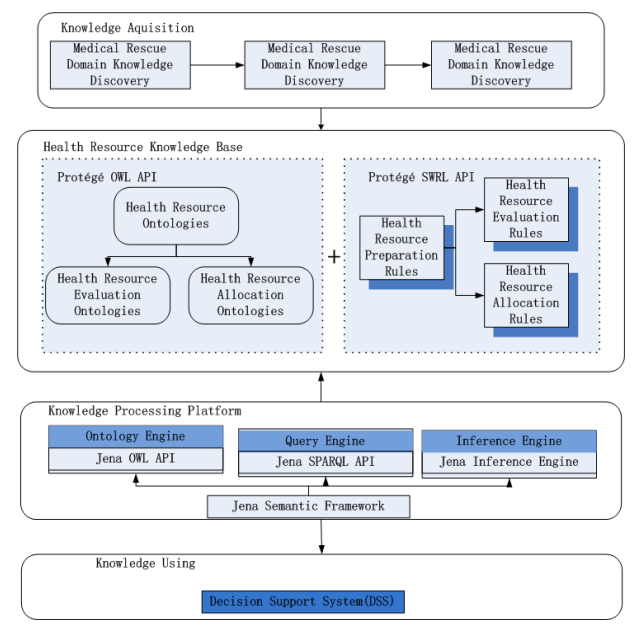

Figure 1. RESEARCH STEPS USED IN THE DEVELOPEMENT OF THE SHRKB validat the method with a case study, and the knowledge base could be used in DSS. All these steps are shown in Figure 1.

\section{DEVELOPMENT OF ONTOLOGY-BASED KNWLEDGE BASE}

\section{A. Knowledge Acquisition}

The knowledge acquisition phase includes three processes: knowledge identification, knowledge extraction, and knowledge authentication. Three types of knowledge sources are identified in the knowledge identification process:

- Published medical literature on healthcare resource preparation plans for crisis rescue (HRPPCR) or medical rescue guidelines, standard nomenclatures and medical rescue information models;

- Healthcare resource data from the history medical rescue tasks;

- Experiences of medical rescue practitioners and domain experts. 1

The define of certain HRPPCR, is to design the type and quantity of the basic and specialized resources based on the possible distribution of the injuries' condition, category and portion injury ${ }^{[16,17]}$. The basic health resources(BHR) are for general injuries which always occur in most crisis rescuing. For example, the health resources for hemostasis, bandaging and fixing. The special health resources(SHR) are for special injuries which occur in certain crisis rescuing. For examples, the medicine and equipments for burned injuries in fire disaster; the medicine used for epidemic disease after flood disaster; etc.

In the processes, we gain a general view of the plan define workflow. We use the UML activity diagram to describe the workflow, as shown in Figure 2(taking BHR preparation as an example).

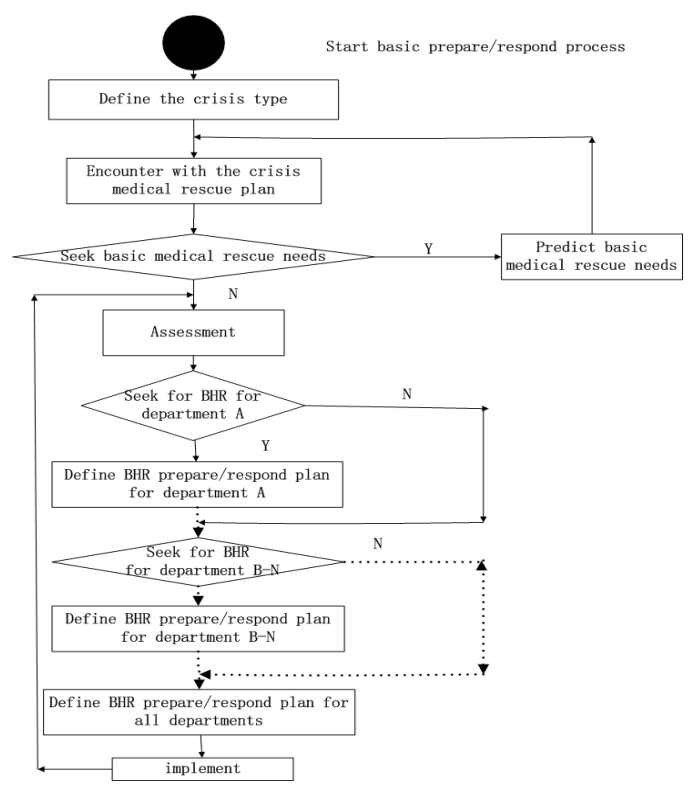

Figure 2. BHR PREPERATION WORKFLOW 
The define of basic HRPPCR is a step-by-step process. Each department of the medical rescue team has a certain function. For example, the triage department mainly responds for firstaiding, sorting and allocating further aids on the basis of medical treatment needs. For another example, the operating department is mainly responded for performing surgery on the injury if necessary. These two departments are of core importance in a medical rescue team and need different types and quantity of health resources based on both the function of the departments and the workloads (medical rescue needs) predicted in the first step. So, we define the basic HRPPCR from department $\mathrm{A}$ to department $\mathrm{N}$ based on the rescue task needs. The whole basic HRPPCR is formed at the end of the process.

\section{B. Information semantic extraction}

We extract information semantics from these class diagrams using the meta-ontology approach ${ }^{[18]}$ : concepts are expressed as classes; attributes and relationships are expressed as properties; decision algorithms are expressed as rules, as shown in Figure 3.

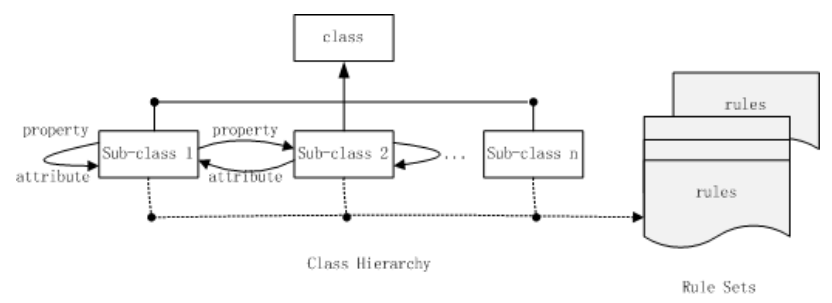

Figure 3. SEMANTIC EXTRACTION USING THE METAONTOLOGY APPROACH

Close consultation with domain experts are involved in the concurrent knowledge authentication process to examine the necessity of extraction and the correctness of extracted information semantics.

\section{Knowledge Representation}

Knowledge representation focuses on the construction of the semantic health resource knowledge base (SHRKB), including the ontology of medical rescue domain knowledge and an expression repository of rules and queries. We develop SHRKB on Protégé, an open-source ontology editor and framework from Stanford University. In this paper, we use the development of the knowledge base of health resource evaluation as an example.

1) Semantic health resource knowledge ontology(SHRKO) SHRKO is the backbone ontology of SHRKB to represent health resource domain knowledge. We develop SHRKO following the ontology developing guide on, main steps include: defining classes, defining the properties of classes, defining the linkages and creating instances.

Following a top-down design pattern, we define four top-level classes in SHRKO, i.e., Data, Model, Expressions and Rules. Below is a brief description of each class: a) 1. Data. Data models the standard data types defined in HL7 Version 3 Data Types Abstract Specification(HL7 v3.0 Data Types Specification.).

b) 2. Model. Model is compliant to the model definition of the HL7 RIM Version 2.41 (HL7 Reference Information Model.), involving three main classes, Act, Role, and Entity, linked together with three association classes: ActRelationship, Participation and RoleLink. Act represents all the acts, performed and recorded by responsible actors in a healthcare workflow; ActRelationship is a directed association between a source act and a target act; Entity represents physical thing, group of physical things or an organization capable of participating in an act while in a role; Role is a competency of an that plays the role as identified, defined, guaranteed, or acknowledged by the entity that scopes the role; Participation is an association between an act and a role; RoleLink is a connection between two roles expressing a dependency between those roles.

c) 3. Expression. Expression is the linkage class between SHRKO and SHRER. We defined the following properties to represent linkage semantics: hasExprId is the expression identifier in SHRKO, hasExprFile is the pointer to the external expression file, hasTriggerSource marks the trigger source of the expression, either time-based, event-based or user defined, hasExpression is a textual description of the expression body.

d) 4. Rule. Rule is the standard to describe the evaluation or distribution of the health resource. We defined the following properties to direct the making of rules in the rule base: Evaluation is the rule to evaluate the resource needs based on the task workloads or the prediction of the wounded types and quantity, Distribution is the rule to determine the health resource's distribution between departments or different medical rescue teams.

Based on this, we take these top-level classes as superclass and constructed subclasses layer by layer. Thus, we describe the conceptions of the sub-node of the knowledge system. Meanwhile, we add relevant attributes and linkages to the subclass. We also reuse existing domain ontologies if necessary, e.g., Systematized Nomenclature of MedicineClinical Terms(SNOMED-CT), Logical Observation Identifiers Names and Codes(LOINC), International Classification of Diseases-10(ICD-10). These are international standard medical term system. Using these ontologies to extend the semantic of our meta ontology could make the ontologies we developed interconnected with the international standards.

we use the development of the knowledge base of health resource evaluation as an example to show the creating of instance.

The objective of constructing the health resource evaluation knowledge base is to provide standards for quick health resource preparation based on different situation. The final plan based on this could be more fit for the mission needing without too much waste. Thus, the medical rescue team could bring more useful health resource with them to save more lives 
making the limited transport capacity to the maxim effectiveness. The main factors to be considered are the number of wounded persons or people in disease, the possible distribution of the different injuries' condition and category, the number of medical rescuer. Health resources largely include medicine(M) resources (mainly pharmaceuticals), medical consumable goods, medical devices and medical instruments. The medicine resources and medical consumable goods are non-reusable resources. Medical devices and medical instruments are reusable resources. The main attributes of the resource were category (Kind) and resource quantity (Number).

Based on the analysis above, we construct the meta ontology to describe the standards of health resource evaluation, including four superclass as Data, Model, Expression and Rule. Based on the meta ontology, we add constraint condition and supplementary condition to reify the subclass. Main subclass include: Medicine Class, describing basic information of medicine, including the attributes of name, ID, Coding System, Frequency, Dosage Unit, Dosage Daily, Drug Interaction, etc.; Device Class, describing basic information of medical devices, including the attributes of name, ID, Coding System, Max workload, Length, Height, etc.; Consumable goods Class, describing basic information of medical consumable goods, including the attributes of name, ID, Coding System, Qualification, etc.; Instrument class, describing basic information of medical instrument, including the attributes of name, ID, Coding System, Function, Assort, etc.; Injury Condition Class, describing specific condition of wounded, including the attributes of injury portion of body, injury type, injury pattern and injury trend; Distribution class, describing specific distribution of the resource between different departments or rescue teams; Term class, including referenced international standard medical term system, e.g. SNOMED-CT, LOINC, ICD-10, etc. Finally, we use OWL to describe the meta ontology and add the ontology instances to form the standard ontology as showed in figure4.

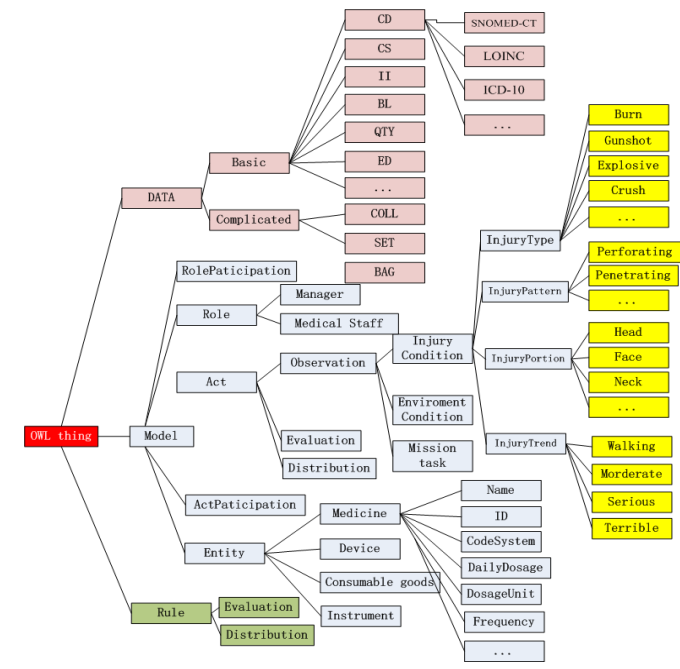

Figure 4. ONTOLOGY MODEL OF HEALTH RESOURCE EVALUATION
In Figure4, we use different color to mark different classes. The yellow marks are instances of certain subclass.

In addition, we import the SWRL temporal ontology to enhance the temporal expressivity of SHRKO with temporal models and built-ins.

2) Semantic health resource expression repository (SHRER)

SHRER is designed to extend SHRKO expressivity with expressions so that it can be queried and reasoned over for knowledge-based decision making. We consider two kinds of expressions: queries and rules. Queries are written in simple protocol and RDF query language (SPARQL). Rules are encoded in Jena rule format.

\section{Knowledge Validation}

We develop a prototype semantic-based health resource preparation decision support system (S-HRPDSS) in Java to enable SHRKB-based preparation decision making. The proposed method is validated in a case study of the overseas humanitarian medical assistance mission executed after a disaster by the hospital ship Peace Ark, a member of China's People's Liberation Army (PLA) Navy.

We instantiate the SHRKB with the domain knowledge from the Chinese marine medical guideline $(\mathrm{CMMG})^{[19]}$ and the standards of medical first aid in marine rescue mission from Advanced first aid afloat (Fifth edition) ${ }^{[20]}$; history marine war medical rescue data contained in the CMMG summaries, electrical medical records from "Mission Harmonious 2011", "Mission Harmonious 2013"and "Mission Harmonious 2014", as well as electrical medical records from emergent department of Navy General Hospital since 2010 are then imported into SHRKO as input fact data; given health resource related data(i.e. injury's or disease's condition, category) and rules from SHRER, we can use the Jena inference engine to derive new facts about the health resource preparation(evaluation or preparation plan recommendations).

Then we use cluster analysis, a statistics method to make plan on certain resource types to cope with the mission needs, based on normal knowledge base and semantic knowledge base to find the differences.

We define the health resource (HR) was defined as: $\mathrm{HR}=(\mathrm{M}$, $\mathrm{C}, \mathrm{D}$ and I ) $\mathrm{M}$ stands for medicine, $\mathrm{C}$ stands for medical consumable goods, D stands for medical Device, I stands for medical instruments. K stands for Kind, the main index in a health resource preparation plan. The cluster analysis is used to determine $\mathrm{K}$ as follows.

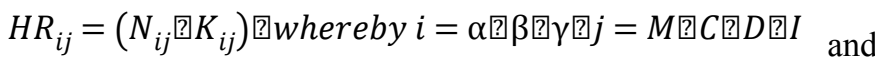
$K_{\alpha}, K_{\beta} \subset K$

$\mathrm{K}_{\mathrm{ij}}$ is to describe all categories of resources; $\mathrm{Nij}$ is the index to describe the quantity of each kind of resources in the $\mathrm{K}_{\mathrm{ij}}$. $\alpha$ is for basic health resources, $\beta$ is for special health resources. $M$ stands for the medicine resources (mainly pharmaceuticals), C stands for medical consumables, D stands for medical devices, 
I stands for medical instruments.

The determination of $\mathrm{K}: \mathrm{K}$ is associated with either the type of crisis or the function of different department in the medical rescue team. So the correlation coefficient $\mathrm{R}$ can be used for resource category clustering. $\mathrm{Xi}$ stands for the frequency use of a certain kind of the resource in " $n$ " samples. When $\mathrm{K}_{\alpha}$ is determined, the samples will be the special health resources used in the rescue task. When $\mathrm{K}_{\beta}$ is determined, the samples will be the health resources used in a certain department of the rescue team. $X_{j}$ stands for the frequency of occurrence of a certain medical rescue tasks in "n" samples. Stronger correlation coefficient reveals a closer link between the resource category and the medical treatment function.

$$
R_{i j}=\frac{\sum_{k=1}^{n}\left(X_{k i}-\bar{X}_{i}\right)\left(X_{k j}-\bar{X}_{j}\right)}{\sqrt{\left[\sum_{k=1}^{n}\left(X_{k i}-\bar{X}_{i}\right) \sum_{k=1}^{n}\left(X_{k i}-\bar{X}_{i}\right)^{2}\right]}}
$$

\section{RESULTS}

A total of 270 classes (4 top-level classes and 266 subclasses), 387 properties (319 object properties and 68 data type properties) and 219 rules are created in SHRKB.

The validation results from the case study of the overseas humanitarian medical assistance mission executed after a disaster by the hospital ship is demonstrated in Table $1 . \mathrm{N}$ stands for the health resource preparation plan clustered by the cluster analysis based on normal medical knowledge base (normal plan for short). S stands for the health resource preparation plan clustered by the cluster analysis based on our semantic health resource knowledge base(SHRKB) (SHRKB plan for short). A stands for the mission actual consumption of the health resource. ( $\mathrm{K}$ stands for the set of health resource categories, $|\mathrm{K}|$ stands for the number of the medical resource categories.)

Table 1- Comparison of the resource categories of normal, actual consumption and SHRKB results. $|K(N)||K(A)||K(S)||K(A) \cap K(N)| \quad \mid K \overline{(A) \cap K(S) \mid}$

\begin{tabular}{llllll}
\hline Consumables & 80 & 56 & 48 & 24 & 8 \\
Medicine & 243 & 167 & 140 & 76 & 27 \\
Equipment & 22 & 20 & 19 & 2 & 1 \\
Instrument & 52 & 52 & 52 & 0 & 0 \\
\hline
\end{tabular}

As it is presented, the resource categories in the normal plan $100 \%$ met the requirement of the actual consumption.

However there were more than $30 \%$ of the non-reusable resource categories in it remained unused during the mission. On the other hand, the inventory of 6 kinds of the non-reusable resources in the normal plan, especially some key resources, would be used up during the mission which indicating that the categories of resources are not adequate for the actual consumption. In the normal plan it appears that the surplus and deficiency could exist side by side.

The categories of the reusable resources in the SHRKB plan meet the requirement of the actual consumption at a more than
$95 \%$ level. The categories of non-reusable resources meet the requirement at a level exceeding $80 \%$ mark. The categories which didn't appear in the plan but is present in the actual consumption are concentrated in some anti-infection medicines or anodyne which are used in a narrow range. These resources could be replaced by others resources which have a wider range of application. The SHRKB plan could meet the actual consumption with less categories of resources. Thus, this plan would be more simple and effective to health resource preparation in emergent case.

From this rough comparison, we could see the results made by the same decision method running on the SHRKB are better. It demonstrates the benefits of the combination use of the HL7 RIM and ontology.

We are still developing a DSS for health resource preparation in marine emergent case. The system prototype is showed in figure 5. This system could help managers to make a quick decision on health resource preparation both in category selecting, quantity calculating, supplement planning and better routes comparing. Thus, the medical rescue team would be ready to set off in a short time and cure injuries in the best time. The right part of the system shows the basic inputs including ship scheme, mission information, departments properties, injuries or disease sets. The up-left part shows the health resource allocation plan in the history case which matches the input condition on the left. The down-left part shows the health resource allocation plan recommended by the system through a series of OM calculating.

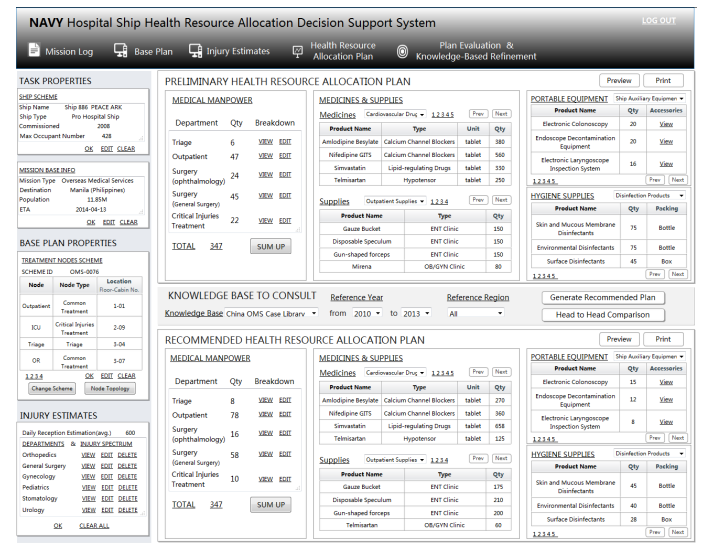

Figure 5 PROTOTYPE OF DSS FOR HEALTH RESOURCE PREPARATION IN EMERGENT CASE.

\section{DISCUSSION AND CONCLUSION}

In this paper, we present a new approach to enhance effective use of DSS in health resource preparation/respond by developing SHRKB, a health resource knowledge base based on rules of HL7 RIM and ontology. HL7 RIM offers a static model of health care information, which includes classes and state-machine diagrams and is accompanied by use case models, interaction models, data type models, terminology models, and other types of models based on which domainspecific information models are then derived through a series of constraining or refinement processes. Ontology provides a 
standard model for data and knowledge representation.

The result of the case study using the same method running on different knowledge base shows, SHRKB could support the method come to more effective plan than normal knowledge base does. This demonstrates our research's advantages as follows: (1) the unified representation formalism for both mission data and domain knowledge to facilitate the semantic interoperability between DSS and healthcare information systems; (2) semantic-based integration and reuse of computer technologies and domain knowledge for convenient knowledge base maintenance; (3) knowledge-based inference for dynamic health resource preparation for crisis relief.

Still, our research has some imperfections. For example, if we want to develop DSS based on SHRKB, the validation of SHRKB is not enough. Considering the complication of the health resource preparation for the crisis, the data and knowledge acquisition are not enough. The evaluating of the health resource preparation plan is not enough. The data and knowledge acquisition is not enough because crisis rescuing is always in a hurry and there's hardly time for the rescuers to collect data or knowledge on purpose.

So, our future work will focus on: (1) develop DSS for health resource preparation based on SHRKB; (2) investigation of knowledge based preparation plan evaluation methods; and (3) integration with machine learning techniques for automatic knowledge acquisition and close-loop knowledge management.

\section{ACKNOWLEDGMENT}

This study is supported by the Project in the National Science \& Technology Pillar Program during the Twelfth Five-year Plan Period (Construction Programming of emergency management mechanism and emergency plan system of towns, Grant No. 2015BAK10B01); Army logistics key projects(\#BHJ12L007, \# CHJ11J016).

\section{REFERENCES}

[1] Maughon JS An inquiry into the nature of wounds resulting in killed inaction in Vietnam. Mil Med, 1970, 1:13

[2] Sikic N, Korac Z, Krajacic I ,et al War abdominal trauma: usefulness of penetrating abdominal trauma index, injury severity score, and number of injured abdominal organs as predictive factors. Mil Med, 2001, 166: 226-230.
[3] Toregas, C., Swain, R.\& ReVelle, C.et al. The Location of Emergency ServiceFacilities. Operations Research, 1971,19(6), 1363-1373.

[4] Badri, M. A.,Mortagy, A. K. \& Alsayed,C. A. A multi-objective model for locating fire stations. European Journal of Operational Research,1998(110),243-260.

[5] Balcik,B. \& Beamon,B. M. Facility location in humanitarian relief. International Journal of Logistics: Research and Applications, 2008, 11(2), 101-121.

[6] Rawls,C. G. \& Tumquist, M. A. Pre-positioning of emergency supplies for disaster response. Transportation Research Part B,2010(44),521-534

[7] Ozbay, K.,Xiao,W. \& lyigun, C.et al. Probabilistic Programming Models for Response Vehicle Dispatching and resource allocation in $\mathrm{t}$ raffic incident. management.I\&SE: Rutgers University, 2004, 4-14.

[8] Karaman, M. M., Elmaghraby, W. \& Salman,F. S. Relief Aid Stocking Decisions under Cooperation of Emergency Response Agencies,2009,14.

[9] Shetty, R. S. An event driven single game solution for resource allocation in a multi-crisis environment. Doctoral Dissertation, University of South Florida,2004.

[10] Gupta, U. Multi-event crisis management using non-cooperative repeated games,Doctoral Dissertation, University of South Florida, 2004.

[11] Sheu, J.B.,Chen, Y. \& Lan, L. W. A novel model for quick response to disaster relief distribution. Proceedings of the Eastern Asia Society for Transportation Studies, 2005 (5), 2454-2462.

[12] Studer R, Benjamins V.R., Fensel D, Knowledge engineering: principles and methods. Data \& Knowledge Engineering, 25(1), pp. 161-197, 1998.

[13] Collier N, Kawazoe A, Jin L, et al. A Multilingual Ontology for Infectious Disease Outbreak Surveillance: Rationale, Design and Challenges[J]. Journal of Language Resources and Evaluation, Springer, 2007,(40):3-4.

[14] Unified Medical Language System Semantic Network Documentation[EB/OL]. 20].http://www.nlm.nih.gov/research/umls/meta3.html

[15] Schadow G, Russler D.C., Mead C.N., et al,Integrating medical information and knowledge in the HL7 RIM. Proc. of the AMIA Symposium, American Medical Informatics Association, pp. 764,2000.

[16] Kaiyu C, Yifeng C, Fen Z. Modularized support of medical supplies in military operations other than war. Academic Journal of Second Military Medical University 2012, 12:1351-1355.(Chinese)

[17] Hui J, Shengxin C, Guoquan R. Demand analysis and application of modularization theory in medical supplies for war preparedness. Academic Journal of Second Military Medical University 2005, 26:815-819.(Chinese)

[18] Z Hu, J.S. Li, T.S. Zhou, H.Y. Yu, Suzuki M, Araki K, Ontology-based clinical pathways with semantic rules. Journal of medical systems,36(4), pp.2203-2212, 2012

[19] Jiyao Yu, Zhengguo Wang. Naval Surgury. People's Military Medical Press, 2014.(Chinese)

[20] Peter F. Eastman, John M. Levinson. Advanced first aid afloat (Fifth edition)[M].Cornell maritime press, Maryland,2004. 